\title{
Determinación del contenido de silicio y cromo en fundiciones grises mediante el método de Van der Pauw ${ }^{(\cdot)}$
}

\author{
E. Tremps*, J.L. Enríquez**, C. Morón*, A. García* y A. Gómez*
}

\begin{abstract}
Resumen
En este trabajo se presenta un sistema basado en la medida de la resistividad de las muestras de la fundición gris mediante el método de Van der Pauw para evaluar el contenido de silicio presente en dichas muestras. Se han realizado 25 ensayos diferentes y se han estudiado las características resistivas y metalográficas de todas las muestras, demostrando que es también posible obtener mediante este método el contenido de silicio en fundiciones con contenidos bajos de aleantes o el contenido de cromo en series de fundiciones en las que la tasa de silicio se mantenga constante y conocida.
\end{abstract}

\section{Determination of silicon and chromium content in gray cast iron by the Van der Pauw method}

\begin{abstract}
In this paper we show a system based on the resistivity measurement of samples of gray cast iron by the Van der Pauw method to calculate the silicon content in the samples. Twenty five trials have been carried out, studying resistive and metallographic characteristics of the samples. This has demonstrated that it is possible to obtain, by this method, the silicon content in molten flat with low content of alloying elements, also the content of chromium in series smelters where the rate of silicon remains constant.
\end{abstract}

Keywords

Cast iron; Resistivity; Silicon content; Van der Pauw method.

\section{INTRODUCCIÓN}

Desde el punto de vista micrográfico, la fundición gris podría considerarse como un acero ferrítico-perlítico en el cual está disperso un retículo continuo de grafito $^{[1]}$. Esta continuidad del grafito rompe, a su vez, la continuidad de la matriz metálica, afectando a propiedades tales como tenacidad, conductividades térmica y eléctrica, etc. Todas estas propiedades son dependientes de los elementos de aleación presentes en la fundición y de la velocidad de enfriamiento.

De estos elementos de aleación, el silicio es el que ejerce más influencia en la estructura y propiedades del material. Los métodos de determinación directa del contenido de silicio de una fundición por vía húmeda han sido sustituidos con ventaja actualmente por la espectrometría de emisión. Sin embargo, las medidas eléctricas pueden ser un método barato e inmediato de evaluar el contenido en silicio en estos materiales.

La conductividad eléctrica de una aleación que consta de dos o más fases depende de las conductividades de estas fases. No obstante, la conductividad de la aleación es una propiedad estructuralmente sensible y depende de la distribución de las fases presentes.

En primer término, al igual que en los sistemas monofásicos, sobre la conductividad eléctrica de la muestra influye el tamaño de grano. Una gran influencia la ejerce también el grado de dispersión para el cual las dimensiones de los pequeños cristales de una de las fases (inclusiones) son del mismo orden de magnitud que la longitud del recorrido libre, es decir, del orden de $1 \mathrm{~nm}$, teniendo lugar además una dispersión complementaria de los electrones en estas inclusiones ${ }^{[2]}$. El efecto máximo de dispersión y, por

(•) Trabajo recibido el día 21 de febrero de 2013 y aceptado en su forma final el día 5 de abril de 2013.

* Dpto. Tec. Edificación, E.U. Arquitectura Técnica (U.P.M.), Madrid 28040, España.

** Dpto. Ingeniería de Materiales, E.T.S.I. Minas (U.P.M.), Madrid 28040, España. 
consiguiente, también el máximo aumento de la resistencia eléctrica corresponden al tamaño de las inclusiones. En este caso el incremento de la resistencia eléctrica puede llegar a ser del $10 \%$ al $15 \%$.

Ejerce también una influencia determinada la textura de la muestra si los cristales pertenecen a un sistema no cúbico. La conductividad eléctrica de una aleación que consta de dos o más fases puede considerarse como una propiedad que se compone aditivamente de las conductividades de dichas fases, si es posible despreciar la influencia de los factores enumerados anteriormente ${ }^{[3]}$.

En principio, es posible calcular la conductividad eléctrica de una aleación basándose en la conductividad de las fases que la integran, si se conoce el contenido volumétrico de cada fase, así como la forma y la disposición relativa de los cristales ${ }^{[4]}$. En este caso, no puede tomarse en consideración la dispersión complementaria de los electrones como resultado de las distorsiones marginales de la red o en inclusiones muy pequeñas.

La conductividad eléctrica efectiva de un compuesto macroscópicamente isótropo con una microestructura arbitraria está comprendida entre dos valores límite ${ }^{[5]}$ (Ec. (1)):

$$
\sigma_{\mathrm{lo}}+\frac{\mathrm{V}_{\mathrm{h}}}{\frac{1}{\sigma_{\mathrm{h}}-\sigma_{\mathrm{lo}}}+\frac{1-\mathrm{V}_{\mathrm{h}}}{3 \sigma_{\mathrm{lo}}}}=\sigma_{\text {lower }} \leq \sigma_{\text {eff }} \leq \sigma_{\text {upper }}=\sigma_{\mathrm{h}}+\frac{1-\mathrm{V}_{\mathrm{h}}}{\frac{1}{\sigma_{\mathrm{lo}}-\sigma_{\mathrm{h}}}+\frac{\mathrm{V}_{\mathrm{h}}}{3 \sigma_{\mathrm{h}}}}(1)
$$

donde $\sigma_{\mathrm{h}}$ y $\mathrm{V}_{\mathrm{h}}$ son la conductividad eléctrica y la fracción de volumen de la fase de más alta conductividad, respectivamente, y $\sigma_{\mathrm{lo}} \mathrm{y} \mathrm{V}_{\mathrm{lo}}$ los mismos valores para la fase de más baja conductividad.

Si estudiamos las fundiciones nodulares ferríticas, hay que tener en cuenta que éstas tienen mayor conductividad térmica que las perlíticas ${ }^{[6]}$. De hecho, la conductividad disminuye cuando la ferrita va desapareciendo. Además, las fundiciones nodulares (grafito esferoidal) tienen menores resistividades eléctricas que las fundiciones grises (grafito laminar) ${ }^{[7 \text { y } 8]}$, y los elementos que más influyen son el silicio y el níquel. En fundición perlítica normal los contenidos de níquel y silicio son del 0,5 \% - 1 \% para el níquel y $2,0 \%-2,5 \%$ para el silicio, siendo la resistividad de aproximadamente $54 \times 10^{-6} \mathrm{ohm} . \mathrm{cm}$. Las resistividades son menores en fundiciones ferríticas, para las que se supone valores de $50 \times 10^{-6} \mathrm{ohm} . \mathrm{cm}$. En la tabla I se comparan valores de diversas fundiciones y aceros.

En cuanto a las propiedades magnéticas, es determinante también la estructura de la matriz. Desde el punto de vista magnético las fundiciones ferríticas son dulces, es decir, su campo magnético de saturación

\section{Tabla I. Valores de la resistividad eléctrica de diversos materiales} Table I. Electrical resistivity values of various
materials

\begin{tabular}{lc} 
Material & $\begin{array}{c}\text { Resistividad } \\
\text { Eléctrica } \\
(\boldsymbol{\mu} \mathbf{\Omega} \mathbf{~ c m})\end{array}$ \\
\hline Fundición nodular, 2\% Cr - 20\% Ni & 102 \\
Fundición laminar al níquel & $130-170$ \\
Fundición gris normal & $75-100$ \\
Acero de medio carbono & 18 \\
Acero al 12\% Cr & 57 \\
Acero inox 18/8 & 70
\end{tabular}

es menor que $1.000 \mathrm{~A} / \mathrm{m}$, y se emplean especialmente cuando se exigen buenas propiedades magnéticas con menores pérdidas magnéticas. Las permeabilidades magnéticas más altas $\left(\mu_{\max }=1.700\right)$ se obtienen en fundiciones exentas de níquel y con muy altos contenidos de silicio, mayores que los normalmente empleados comercialmente.

Si tenemos en cuenta las fundiciones nodulares ${ }^{[2 \mathrm{y} 9]}$, donde todo su grafito en forma esferoidal se encuentra disperso en una matriz casi siempre predominantemente ferrítica, la conductividad térmica dependerá del contenido en silicio. Así, si aumenta el contenido de silicio del $2 \%$ al $3 \%$, la conductividad térmica decrecerá un $20 \%$. Si se estudia la variación de la resistividad eléctrica con el contenido de silicio a temperatura ambiente, se puede observar cómo aumenta casi un $20 \%$ al pasar de $2,5 \%$ a $4 \%$ dicho contenido (Tabla II).

De la misma forma, realizando el estudio de las propiedades magnéticas resulta que un aumento del contenido de silicio desde el 2,0 \% al 3,0 \% duplica la permeabilidad magnética.

Si se tienen en cuenta ahora los distintos valores de la resistividad que presentan los componentes de

Tabla II. Variación de la resistividad en función del contenido de silicio

Table II. Resistivity variation in depending on the silicon content

\begin{tabular}{cc}
\hline Si (\%) & Resistividad $\left(\mu \Omega / \mathbf{c m}^{3}\right)$ \\
\hline 2,5 & 57,8 \\
3,0 & 66,5 \\
4,0 & 72,7 \\
\hline
\end{tabular}


la fundición gris (Tabla III), en este trabajo se ha realizado el estudio de la resistividad eléctrica de un conjunto de muestras de composición y estructura usuales en el campo de una fundición industrial, con el fin de poder extraer una correlación entre resistividad y composición.

\section{MÉTODO EXPERIMENTAL}

\subsection{Toma de muestras}

Las aleaciones ensayadas se han elaborado en un horno de cubilote situado en una planta de fundición industrial dedicada a la producción de piezas moldeadas destinadas a construcción mecánica, automoción, valvulería y otras aplicaciones.

Las fundiciones obtenidas en cubilote se cuelan en unos moldes cilíndricos, elaborados con arena sílice aglutinada con bentonita. No se cuela en molde metálico, como parecería más cómodo y rápido, porque la colada en molde de arena garantiza velocidad de enfriamiento constante en todos los ensayos. De esta forma, se fija esta variable y se eliminan en su mayor parte las variaciones micro-estructurales y de propiedades que originaría.

Una vez solidificado y frío el metal colado, se rompe el molde. Se obtiene de esta forma una barra cilíndrica de $500 \mathrm{~mm}$ de longitud y $20 \mathrm{~mm}$ de diámetro, que se granalla para eliminar la arena adherida a su superficie. Simultáneamente con las piezas, y con el mismo metal, se cuelan las muestras para análisis químico por espectrometría de emisión.

\subsection{Medida de la resistividad}

Siguiendo la sistemática descrita en el epígrafe anterior se tomaron 25 muestras del horno de cubilote durante su marcha normal en la planta de fundición. La composición de dichas muestras se detalla en la tabla IV.

A fin de medir la resistividad eléctrica se utilizó el método de Van der Pauw (método de las cuatro puntas ${ }^{[10]}$, haciendo pasar por la muestra una corriente de 3 A de intensidad y midiendo la tensión mediante un multímetro entre dos contactos situados a $400 \mathrm{~mm}$ de separación. El multímetro utilizado es un Hameg HM 8112-2 cuya precisión en la medida del voltaje en la escala de $200 \mathrm{mV}$ es de 0,002 \% de la lectura más el 0,0007 \% del fondo de escala. Los datos obtenidos se muestran en la tabla IV.
Tabla III. Valores de la resistividad de los componentes de la fundición gris

Table III. Resistivity values of the gray iron components

Dado que la resistencia de un conductor viene dada por la expresión (Ec. (2)):

$$
R=\rho \frac{L}{S}
$$

y que dicha resistencia según la ley de $\mathrm{Ohm}$ viene dada por la ecuación (3):

$$
R=\frac{U}{I}
$$

es evidente que, (Ec. (4)):

$$
\rho=R \frac{S}{L}=\frac{S \cdot U}{L \cdot I}=k U
$$

En otras palabras, como los valores de $S, L$ e $I$ han sido los mismos para todas las muestras, los valores obtenidos de $U$ serán directamente proporcionales a la resistividad de la muestra. Como estos valores son más manejables, se ha preferido trabajar con ellos en los cálculos siguientes.

\section{RESULTADOS}

Los resultados obtenidos tanto de composición como de voltaje se dan en la tabla IV. Las muestras 1 y 8 eran de fundición nodular (grafito esferoidal), presentando las resistividades más bajas del conjunto.

Dichas muestras han sido preparadas metalográficamente y examinadas mediante un micros- 
DETERMINACIÓN DEL CONTENIDO DE SILICIO Y CROMO EN FUNDICIONES GRISES MEDIANTE EL MÉTODO DE VAN DER PAUW DETERMINATION OF SILICON AND CHROMIUM CONTENT IN GRAY CAST IRON BY THE VAN DER PAUW METHOD

Tabla IV. Composición química de las muestras de fundición de hierro obtenidas mediante espectrometría de emisión

Table IV. Chemical composition of the cast iron samples obtained through emission spectrometry

\begin{tabular}{ccccccccccc}
\hline Muestra & $\% \mathbf{C}$ & $\% \mathbf{S i}$ & $\% \mathbf{M n}$ & $\% \mathbf{S}$ & $\% \mathbf{P}$ & $\% \mathbf{C r}$ & $\% \mathbf{N i}$ & $\% \mathbf{M o}$ & $\% \mathbf{C u}$ & $\mathbf{U}(\mathbf{m V})$ \\
\hline 1 & 3,6 & 2,5 & 0,13 & 0,04 & 0,07 & 0,035 & 0,034 & $<0,01$ & 0,10 & 2,06 \\
2 & 3,4 & 2,0 & 0,39 & 0,19 & 0,31 & 0,075 & 0,053 & $<0,01$ & 0,24 & 3,34 \\
3 & 3,4 & 2,0 & 0,38 & 0,15 & 0,28 & 0,096 & 0,073 & $<0,01$ & 0,20 & 3,13 \\
4 & 3,8 & 2,4 & 0,37 & 0,21 & 0,11 & 0,23 & 0,180 & 0,050 & 0,37 & 3,78 \\
5 & 3,5 & 2,4 & 0,38 & 0,19 & 0,12 & 0,23 & 0,210 & 0,050 & 0,46 & 3,5 \\
6 & 3,6 & 2,6 & 0,39 & 0,18 & 0,12 & 0,18 & 0,160 & 0,040 & 0,36 & 4,04 \\
7 & 3,4 & 2,1 & 0,37 & 0,15 & 0,09 & 0,23 & 0,120 & 0,050 & 0,27 & 3,01 \\
8 & 4,1 & 2,1 & 0,10 & 0,02 & 0,06 & $<0,01$ & 0,030 & $<0,01$ & 0,07 & 2,26 \\
9 & 3,3 & 2,5 & 0,39 & 0,18 & 0,13 & 0,20 & 0,150 & 0,030 & 0,43 & 3,61 \\
10 & 3,4 & 2,2 & 0,52 & 0,13 & 0,11 & 0,29 & 0,250 & 0,041 & 0,43 & 3,32 \\
11 & 3,4 & 2,2 & 0,53 & 0,12 & 0,10 & 0,28 & 0,190 & 0,050 & 0,41 & 3,07 \\
12 & 3,4 & 2,0 & 0,55 & 0,13 & 0,09 & 0,38 & 0,260 & 0,070 & 0,34 & 2,9 \\
13 & 3,4 & 2,3 & 0,47 & 0,15 & 0,09 & 0,20 & 0,075 & 0,029 & 0,23 & 3,59 \\
14 & 3,4 & 2,1 & 0,51 & 0,13 & 0,09 & 0,20 & 0,072 & 0,025 & 0,20 & 3,09 \\
15 & 3,4 & 2,1 & 0,48 & 0,15 & 0,08 & 0,32 & 0,070 & 0,020 & 0,19 & 2,98 \\
16 & 3,4 & 2,0 & 0,49 & 0,15 & 0,08 & 0,23 & 0,075 & 0,020 & 0,20 & 3,18 \\
17 & 3,5 & 2,2 & 0,45 & 0,14 & 0,10 & 0,19 & 0,071 & 0,020 & 0,23 & 3,34 \\
18 & 3,4 & 2,2 & 0,50 & 0,12 & 0,11 & 0,17 & 0,073 & 0,030 & 0,85 & 3,58 \\
19 & 3,4 & 2,4 & 0,50 & 0,12 & 0,13 & 0,17 & 0,084 & 0,050 & 0,80 & 3,96 \\
20 & 3,4 & 2,3 & 0,43 & 0,13 & 0,14 & 0,15 & 0,075 & 0,030 & 0,54 & 3,58 \\
21 & 3,4 & 2,6 & 0,39 & 0,13 & 0,10 & 0,14 & 0,065 & 0,030 & 0,40 & 4,18 \\
22 & 3,4 & 2,4 & 0,39 & 0,17 & 0,14 & 0,15 & 0,071 & 0,030 & 1,15 & 3,6 \\
23 & 3,4 & 2,3 & 0,41 & 0,18 & 0,18 & 0,11 & 0,066 & 0,020 & 1,20 & 3,61 \\
24 & 3,4 & 2,3 & 0,40 & 0,16 & 0,16 & 0,16 & 0,070 & 0,030 & 0,87 & 3,8 \\
25 & 3,4 & 2,2 & 0,40 & 0,15 & 0,11 & 0,23 & 0,074 & 0,040 & 0,41 & 3,3 \\
\hline
\end{tabular}

copio óptico. En todas las medidas del tamaño de partículas se ha seguido la norma UNE 36-117. De esta forma se ha obtenido que en la muestra 1 el núcleo de la barra de grafito es laminar y la distribución es del tipo A. Además, el tamaño de las partículas de grafito es el 4 (Fig. 1 (a)). Se ha realizado el estudio superficial obteniendo que en esta muestra la forma del grafito también es laminar, y la distribución es mezcla de los tipos A y B (en rosetas), siendo el tamaño de las partículas de grafito de 4/5 (Fig. 1 (b)). De la misma manera, la microestructura de la matriz metálica es perlita laminar fina y algunas partículas de eutéctico fosforoso (Fig. 1 (c)).

En la muestra 8 el grafito es esferoidal en toda la sección transversal de la barra (Fig. 2 (a)), el tamaño de los nódulos de grafito es el 6 y la microestructura de la matriz metálica es perlita laminar fina y ferrita en el entorno de los esferoides de grafito, como se puede observar en la figura 2 (b).
El resto de las muestras eran de fundición laminar y con ellas se procedió a realizar el estudio estadístico.

\subsection{Análisis de regresión}

Se procedió a realizar análisis de regresión del voltaje obtenido, $U$, frente a composición. Los mejores resultados se obtienen al analizar el voltaje frente a los contenidos de silicio y cromo. La ecuación resultante es, (Ec. (5)):

$$
\mathrm{U}=0,336+1,528 \cdot \mathrm{Si}-1,6 \cdot \mathrm{Cr}
$$

Los datos correspondientes a la regresión se pueden observar en la tabla V.

El coeficiente de determinación tiene el valor 0,85 , lo que en principio significa que un $85 \%$ de la 


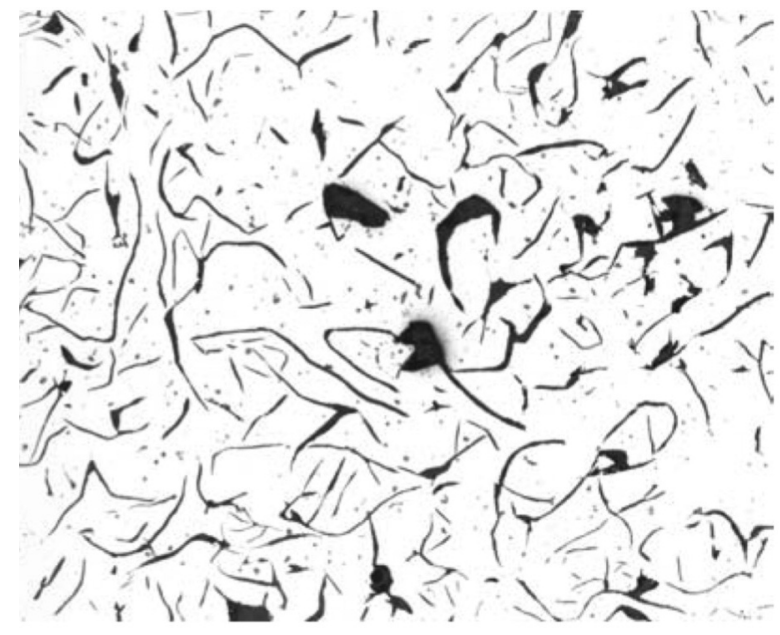

(a)

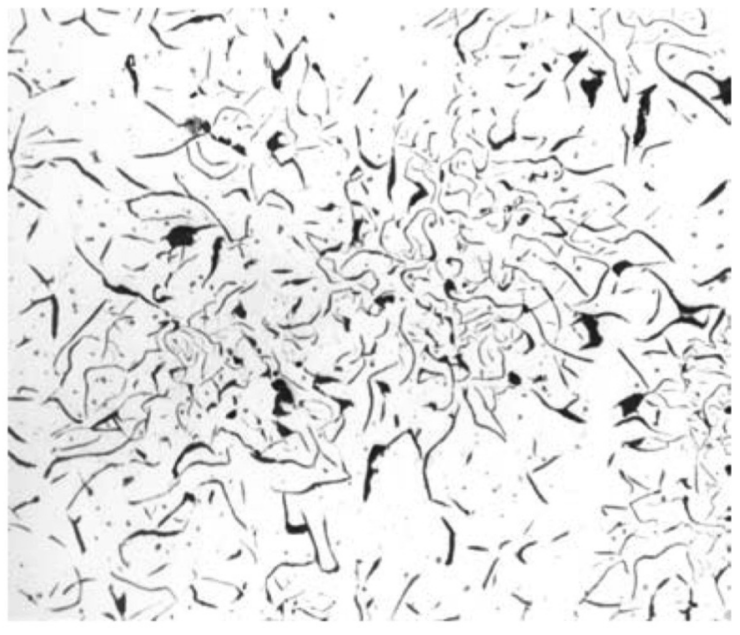

(b)

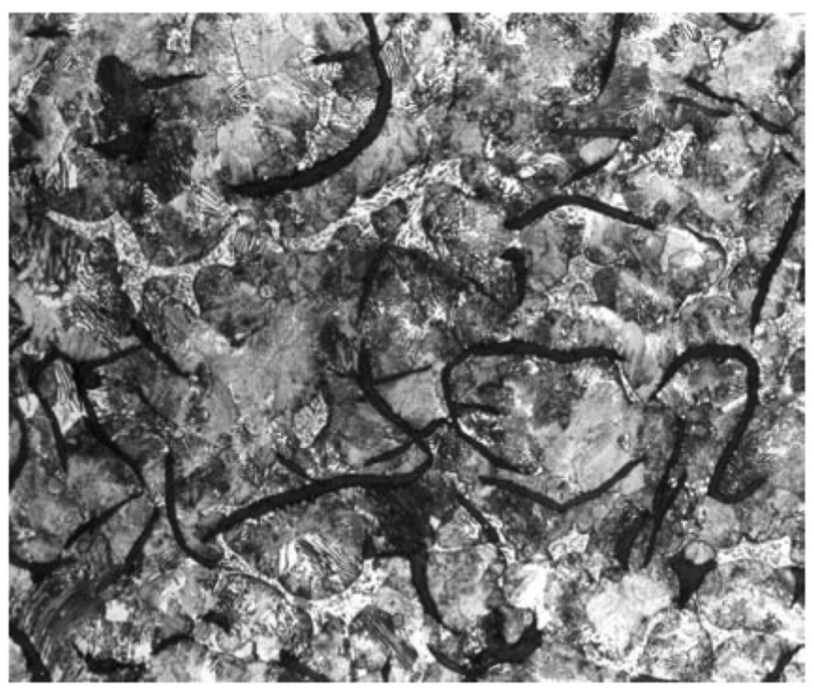

(c)

Figura 1. Estudio metalográfico de la muestra 1: (a) núcleos; (b) superficie de la barra de grafito y (c) matriz metálica de perlita laminar fina.

Figure 1. Metallographic study of sample 1: (a) nucleus; (b) the graphite rod surface and (c) metal matrix of fine laminar pearlite.

variabilidad de $U$ es función de los contenidos en cromo y silicio.

El test de la "F" es alto, 58,3 con un p-valor 4,53E-09, lo que indica que el modelo es consistente en cuanto a la dependencia de $U$ respecto a los contenidos de silicio y cromo.

Por lo que respecta a cada elemento por separado, el elemento más influyente es el silicio, ya que la $t$ de Student para su coeficiente es 9,03 con un p-valor de 1,7012E-08.

Para el coeficiente del cromo se tiene una $t$ de Student de $-3,70$ con p-valor de 0,001413. Por tanto, la dependencia del cromo también es significativa, aunque menor que la del silicio.

A continuación se estudiará la linealidad del modelo analizando los residuos, que son la diferencia entre el valor previsto y el obtenido. Es decir, (Ec. (6)):

$$
e_{j}=U_{j}-\left[0,336+1,528 \cdot(S i)_{j}-1,6 \cdot(C r)_{j}\right]
$$

El gráfico de residuos se muestra en la figura 3 (a). A simple vista no se observa curvatura, ni nada que 


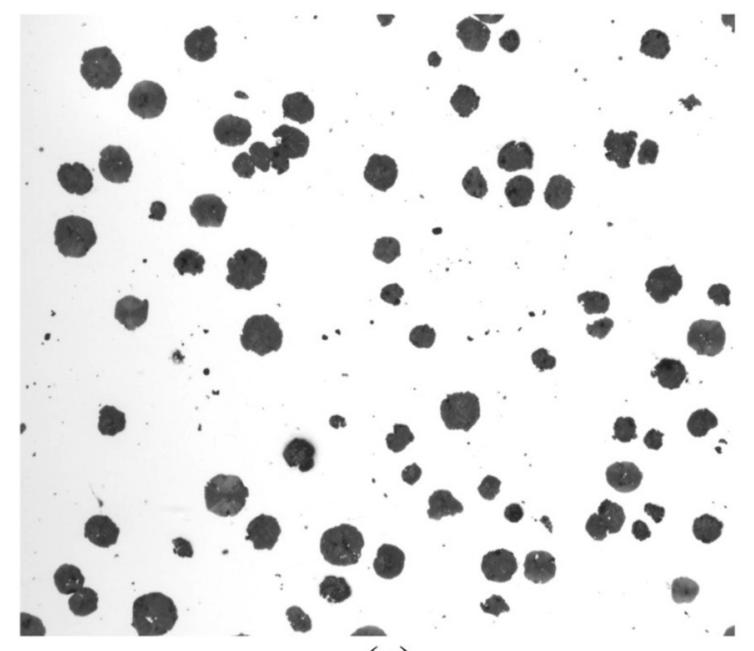

(a)

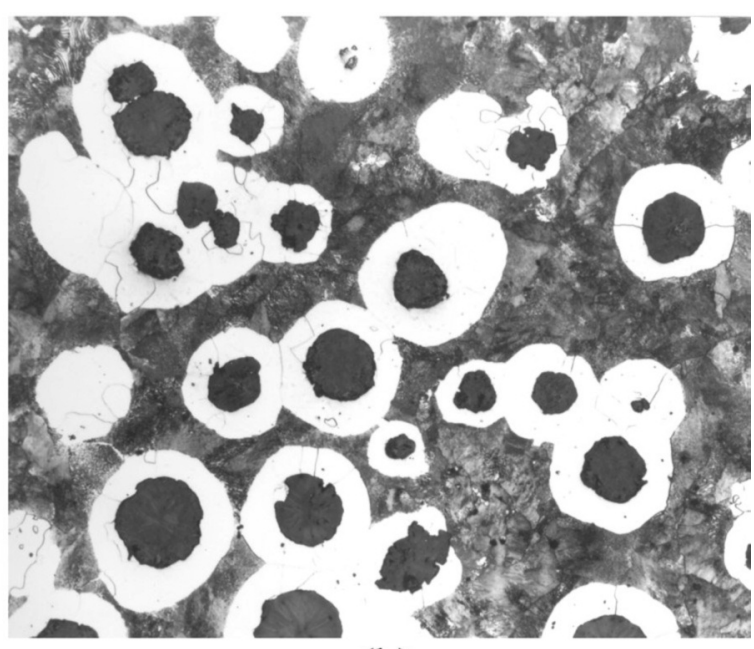

(b)

Figura 2. Estudio metalográfico de la muestra 8: (a) sección transversal de la barra y (b) matriz metálica (b).

Figure 2. Metallographic study of the sample 8: (a) cross section of the bar and (b) metal matrix.

Tabla V. Datos obtenidos de la regresión. La variable X1 corresponde al silicio y la X2 al cromo

Table V. Data obtained from the regression. Corresponds the variable X1 with silicon and variable $X 2$ with chromium

\section{Estadísticas de la regresión}

Coeficiente de correlación múltiple Coeficiente de determinación $\mathrm{R}^{2}$

$\mathrm{R}^{2}$ ajustado

Error típico

Observaciones
0,923895932
0,853583692
0,838942062
0,141400951

23

Análisis de varianza

\begin{tabular}{lcccccc}
\hline & $\begin{array}{c}\text { Grados de } \\
\text { libertad }\end{array}$ & $\begin{array}{c}\text { Suma de } \\
\text { cuadrados }\end{array}$ & $\begin{array}{c}\text { Promedio de los } \\
\text { cuadrados }\end{array}$ & F & $\begin{array}{c}\text { Valor crítico } \\
\text { de F }\end{array}$ \\
\hline $\begin{array}{l}\text { Regresión } \\
\text { Residuos }\end{array}$ & 2 & 2,331263246 & 1,165631623 & 58,2984031 & $4,52788 \mathrm{E}-09$ \\
Total & 22 & $\begin{array}{l}0,399884581 \\
2,731147826\end{array}$ & 0,019994229 & & & \\
\hline & Coeficientes & Error & Estadístico & Probabilidad & Inferior & Superior \\
& & típico & $\mathbf{t}$ & & $\mathbf{9 5} \%$ & $\mathbf{9 5} \%$ \\
\hline Intercepción & 0,336457173 & 0,410505753 & 0,81961622 & 0,4220954 & $-0,519842819$ & 1,19275717 \\
Variable X 1 & 1,527606738 & 0,169121196 & 9,032615513 & $1,7012 \mathrm{E}-08$ & 1,174826106 & \\
Variable X 2 & $-1,600204733$ & 0,432338298 & $-3,701279164$ & 0,001413 & $-2,502046615$ & \\
\hline
\end{tabular}


indique desviación de la linealidad. Asimismo se observa homocedasticidad. Esto se ve confirmado por el gráfico de normalidad de residuos (Fig. 3 (b)), donde se aprecia que están dentro de la hipótesis de normalidad.

\subsection{Discusión}

Puede sorprender que se haya limitado el modelo de la resistividad al contenido de silicio y cromo dejando de lado los otros componentes. Evidentemente se ha visto que el coeficiente de determinación era $85 \%$, por lo que hay un $15 \%$ de variabilidad no explicada por el modelo, en el que se incluyen el resto de componentes y otros factores.

En lo que concierne a los contenidos de manganeso, níquel, fósforo y azufre puede comprobarse que son relativamente bajos, por lo que su influencia a la hora de estudiar la resistividad de las muestras es muy escasa. Por lo tanto, hay que limitar la validez del modelo a fundiciones con contenidos iguales o menores que éstos.

Aunque los contenidos en cobre son mayores, tampoco parece tener una influencia apreciable. Este razonamiento no es válido para el carbono y puede extrañar su ausencia en el modelo.

El contenido en carbono de la fundición no dice nada relativo a la forma en que se presenta. Una parte del mismo se encuentra en forma de grafito, otra en forma de cementita en las láminas de perlita, una parte disuelto en la ferrita y otra parte como cementita. Al investigar la resistividad del hierro con muy pequeños contenidos de carbono se ha obtenido que hasta $0,02 \%$ de $\mathrm{C}$ la resistividad del hierro crece con mucha mayor rapidez que para contenidos más altos. Este hecho se explica porque la solubilidad del carbono en Fe-alfa tiene el máximo en el 0,02 \% de C. Por consiguiente, como en el caso de la fundición este valor se sobrepasa ampliamente, se concluye que la resistividad de la ferrita es un factor constante en la resistividad total. La cementita es un compuesto químico cuya resistividad nada tiene que ver con la del carbono, y lo mismo ocurre con la de la perlita. Por consiguiente, la relación contenido de carbonoresistividad es difícil de establecer. Además, hay que tener en cuenta que el carbono es sensiblemente constante $(3-3,5 \%)$ en la práctica de fundición por lo que la posible influencia de sus variaciones es despreciable. Por lo que se refiere al grafito su influencia en la resistividad se debe más a su disposición y tamaño que a su contenido. Realmente el efecto producido es una desviación en el recorrido de los electrones a lo largo de la muestra. Este factor es muy difícil de cuantificar.

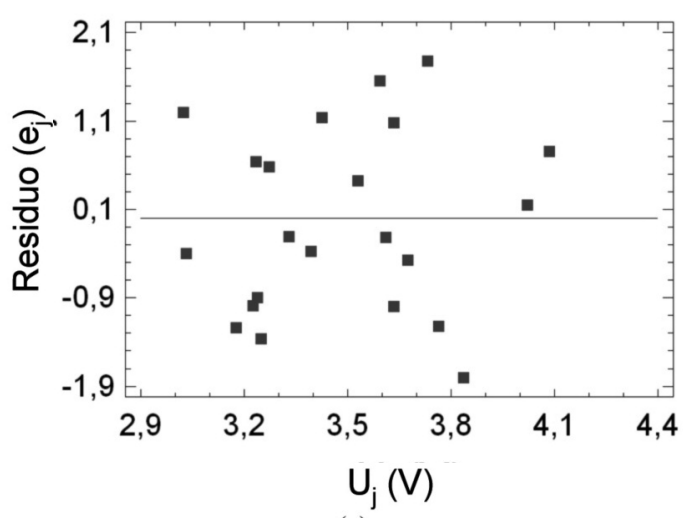

(a)

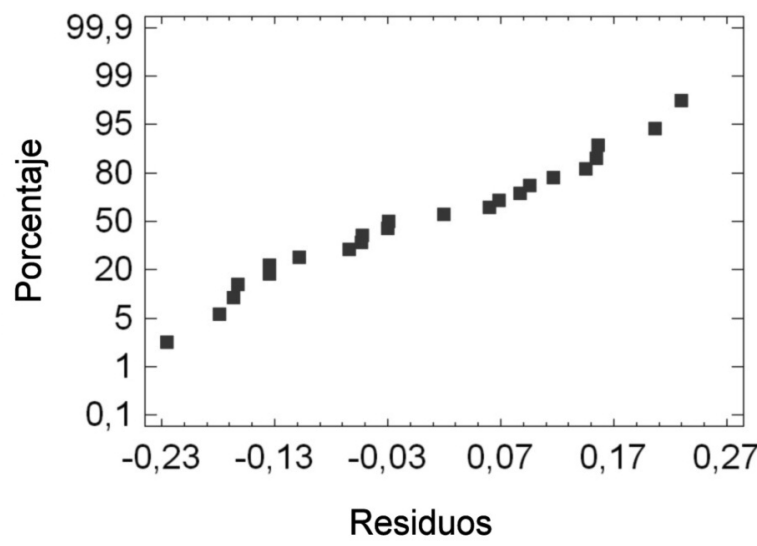

(b)

Figura 3. Estudio de residuos: (a) gráfico de residuos y (b) gráfico de normalidad.

Figure 3. Study of residues: (a) residues graph and (b) graph of normality.

Para terminar no hay que olvidar que el elemento mayoritario en la fundición es el hierro, que es también el máximo responsable de la conducción eléctrica. Por tanto lo que más influye en la resistividad de la fundición es la presencia de aquellos elementos que alteren la resistividad del hierro.

Pese a que los contenidos en cromo también son bajos, éste parece influir más que los otros elementos. Es sabido que el cromo tiene un efecto contrario al silicio en lo referente a la grafitización y que estabiliza los carburos a alta temperatura, por lo que parece lógico que su coeficiente en la ecuación de regresión aparezca con signo negativo, disminuyendo la resistividad.

Por lo que respecta al silicio, es sabido que en las soluciones sólidas Fe-Si el silicio no se comporta idealmente. Los átomos de silicio están más fuertemente asociados con los átomos de hierro que con otros átomos de silicio, reduciendo así la actividad química de éste por debajo de la predicción 
de comportamiento ideal. La fuerte asociación entre átomos de silicio y de hierro está indicada por dos hechos:

Se libera energía (calor exotérmico de solución) cuando se adiciona silicio al hierro líquido.

Se forman fácilmente compuestos intermetálicos de hierro y silicio en fase sólida.

Esto verifica lo apuntado al decir que el silicio al disolverse en la ferrita disminuye la conductividad de la fundición. Es evidente que la resistividad eléctrica puede verse influida por otros factores: tratamientos esferoidizantes, velocidad de enfriamiento en el molde, tratamientos térmicos posteriores, etc. El estudio de la resistividad eléctrica en estos casos, podría ser objeto de trabajos posteriores.

\section{CONCLUSIONES}

La discusión de resultados obtenidos a lo largo de este trabajo ha permitido llegar a las siguientes conclusiones:

- El conjunto de composiciones estudiadas corresponde a fundiciones grises de baja o media aleación, utilizadas en construcción mecánica, a las que no se exige unas características especiales.

- Para el rango de composiciones ensayadas, el elemento más influyente en la resistividad eléctrica es el silicio.

- Con rangos de composición y estructura limitados, la resistividad eléctrica puede ser un buen parámetro para estimar la cantidad de silicio presente.

- De los elementos presentes, sólo silicio y cromo ejercen una influencia sustancial sobre la conductividad de las fundiciones. En base a ello, la medida de la conductividad eléctrica de muestras de fundición podría emplearse:

- Para determinar el contenido de silicio en fun- diciones no aleadas o con contenidos muy bajos de aleantes, especialmente cromo.

- Para determinar el contenido de cromo en series de fundiciones en las que la tasa de silicio se mantiene constante.

- El método puede tener interés adaptándolo a grandes series de piezas donde se diseñe "ad hoc" un método de medida. En estos casos, las medidas de resistividad eléctrica pueden dar información de desviaciones de composición y/o estructura, lo que puede aplicarse al control de calidad de la fundición.

\section{REFERENCIAS}

[1] E. Fras, M. Górny y H.F. López, Metallurgy and Foundry Engineering 31 (2005) 113-116 y 137 147.

[2] S.I. Karsay, Ductile Iron II, Engineering Design Properties Applications, Eds. Quebec Iron and Titanium Corporation, Canadá, 1971.

[3] A. Pazourek, W. Pfeiler y V. Síma, Intermetallics 18 (2010) 1.303-1305.

[4] T. Uchimoto, T. Takagi y T. Abe, Mater. Trans. 51 (2010) 1.114-1.119.

[5] A. Velichko, A. Wiegmann y F. Mücklich, Acta Mater. 57 (2009) 5.023-5.035.

[6] A.F. Spengler, The Ductile Iron Process, Compendium V, Ed. Miller and Company, Chicago Illinois, EE.UU., 1976.

[7] F. Carreño, M. Pozuelo, J. Chao y O.A. Ruano, Rev. Metal. 37 (2001) 130-134.

[8] P. Larrañaga y J. Sertucha, Rev. Metal. 46 (2010) 370-380.

[9] R. Castillo, V. Bermont y V. Martinez, Rev. Metal. 35 (1999) 329-334.

[10] J. Náhlík, I. Kasparková y P. Fitl, Measurement 44 (2011) 1.968-1.979. 